\title{
Alcohol Withdrawal Syndrome in Trauma Patients: A Prospective Cohort Study
}

\author{
Brian Sharp ${ }^{1}$, Carol R. Schermer ${ }^{2}$, Thomas J. Esposito*2, Ellen C. Omi ${ }^{3}$, Hieu Ton-That ${ }^{2}$ and John M. Santaniello ${ }^{2}$
}

${ }^{1}$ University of Michigan Medical Center, Loyola University Medical Center, USA

${ }^{2}$ Loyola University Burn \& Shock Trauma Institute, Loyola University Medical Center, USA

${ }^{3}$ Advocate Christ Medical Center, Loyola University Medical Center, USA

\begin{abstract}
Introduction: Trauma patients with a positive blood alcohol concentration (BAC) are often believed to be at high risk for the alcohol withdrawal syndrome (AWS). Therefore some centers prophylaxis all BAC positive patients. This study prospectively measures the incidence of AWS among trauma patients admitted to the hospital who have consumed alcohol and determines their risk factors for AWS.
\end{abstract}

Methods: A cohort of trauma patients admitted to a non-ICU hospital setting was prospectively monitored for the development of AWS during the first 10 days of hospitalization. The 10-item Alcohol Use Disorders Identification Test (AUDIT) and questions about alcohol withdrawal history were administered on the first day and the revised Clinical Institute for Withdrawal of Alcohol Scale (CIWA-Ar) was administered daily.

Results: 113 patients were followed through discharge or for the first 10 days of hospitalization. $74.3 \%(n=84)$ reported drinking alcohol. Of the 89 patients with a measured BAC, $25(28 \%)$ were positive. Mean BAC for positive patients was $187.7 \mathrm{mg} / \mathrm{dl}$. No person who denied drinking had a measurable BAC or developed AWS. Among the 84 drinkers, 3 were diagnosed with AWS by CIWA-Ar (3.6\% risk), giving an incidence rate of 1.4 episodes per 100 patient days. All patients developing AWS admitted to a previous history of AWS symptoms upon stopping drinking. All AWS patients drank at least 2-3 times per week compared to only $37 \%$ of drinkers who did not develop AWS (p $=.05$ ). Positive response to dependence items from the AUDIT were highly associated with AWS risk (67\% AWS vs $16 \%$ non-AWS, $p=.005$ ). Implementation of a prophylaxis protocol for all positive BAC would have resulted in $88 \%$ $(22 / 25)$ of BAC positive patients receiving unwarranted medication.

Conclusion: AWS has a low incidence rate among intoxicated trauma patients admitted to a non-ICU setting. It is associated with frequent drinking and is found in patients who report dependence symptoms. Patients can reliably tell physicians whether they are at risk for AWS. Routine prophylaxis for positive BAC patients will likely result in substantial excess medication use.

Keywords: Alcohol withdrawal syndrome; Alcohol withdrawal prophylaxis; Trauma patients \& Alcohol

Abbreviations: AUDIT: Alcohol Use Disorders Identification Test; AWS: Alcohol Withdrawal Syndrome; BAC: Blood Alcohol Concentration; CIWA: Clinical Institute for Withdrawal of Alcohol; ICU: Intensive Care Unit

\section{Introduction}

Alcohol use is prevalent in trauma patients, with nearly $50 \%$ of admitted trauma patients having a positive blood alcohol concentration (BAC) upon admission [1,2]. This is generally considered to be any concentration greater than $0 \mathrm{mg} / \mathrm{dl}$. One consideration when caring for patients who misuse alcohol is the risk of alcohol withdrawal. The alcohol withdrawal syndrome (AWS) is due to the rapid removal of the depressant effects of alcohol on the central nervous system. AWS occurs when a patient who is physiologically dependent on alcohol ceases or severely reduces alcohol consumption [3-5]. Because the prevalence of alcohol use disorders in trauma patients is high, it is conceivable that their risk for AWS is also high and could complicate their hospitalization for injury. However, the incidence of AWS among trauma patients has not been previously prospectively evaluated.

Studies in non-trauma patients have described several different risk factors for AWS including amount of alcohol intake, the duration of a patient's drinking habit, history of AWS, older age, and abnormal liver function [4]. One study in trauma patients has demonstrated age, race, or mechanism of injury as risk factors for delirium tremens
[1]. Common symptoms of alcohol withdrawal include anxiety, sleep disturbances, headache, nausea, vomiting, and irritability with some people experiencing tachycardia, sweating, tremulousness and disorientation. In its severe form, delirium tremens may occur with agitation, fever, confusion, hallucinations, and seizures. The majority of patients with AWS experience 24-48 hours of mild to moderate symptoms. However, some patients will develop delirium tremens, which carries a $15 \%$ mortality if left untreated, but can be reduced to $1-5 \%$ with treatment $[3,4]$.

The primary treatment recommendations for AWS from the American Society of Addiction Medicine include benzodiazepines, with carbamazepine, haloperidol, and beta blockers added as adjunctive therapy [4]. Some trauma centers attempt to avoid the development of AWS by providing prophylaxis based on admission BAC and other trauma centers use a fixed drug schedule for prophylaxis of intoxicated

${ }^{*}$ Corresponding author: Thomas J. Esposito, Loyola University Medical Center, 2160 South 1st Ave Building 110, Room 3276 Maywood, IL 60153 USA, Tel: 708-327-2072; Fax: 708-327-3474; E-mail: tesposi@lumc.edu

Received February 21, 2012; Accepted April 13, 2012; Published April 16, 2012

Citation: Sharp B, Schermer CR, Esposito TJ, Omi EC, Ton-That H, et al (2012 Alcohol Withdrawal Syndrome in Trauma Patients: A Prospective Cohort Study. J Trauma Treat 1:128. doi:10.4172/2167-1222.1000128

Copyright: (c) 2012 Sharp B. This is an open-access article distributed under the terms of the Creative Commons Attribution License, which permits unrestricted use, distribution, and reproduction in any medium, provided the original author and source are credited. 
patients to avoid AWS [6] if the patient is deemed to be at high risk for the alcohol withdrawal syndrome, a judgment often based on the admission BAC. However, symptom triggered therapy, rather than prophylaxis for AWS in at-risk patients has been shown to lead to shorter duration of treatment and less overall drug use [4,7-10].

The purpose of this study was to determine the risk factors for AWS among trauma patients admitted to the hospital. The hypotheses of the study were that the risk for alcohol withdrawal would be low and that it could be predicted by simple questioning, thus avoiding potentially unnecessary prophylaxis. Establishing the incidence rate and predictive factors allows for proper identification and timely initiation of treatment in patients with AWS while avoiding unwarranted medication of BAC positive patients who are not at risk for the syndrome.

\section{Materials and Methods}

A cohort of patients admitted to the hospital following blunt or penetrating trauma was prospectively followed for the development of AWS during the first 10 days of hospitalization. The study was approved by the institutional review board and was conducted at a Level I trauma center over nine months. Patients included for study were English speaking individuals, 18 years and older admitted to the hospital in a non-ICU setting. Per institutional protocol, in order to be admitted to a non-ICU bed, patients needed to have no evidence of traumatic brain injury on admission either by CT scan or Glasgow Coma Scale (GCS) score, and have injuries that did not result in hemodynamic compromise. Patients admitted to the floor with a GCS of 14 were only included if the GCS had normalized to 15 by the time of informed consent. Specifically excluded were non English speaking patients, and those admitted directly to the operating room or ICU from the emergency department due to brain injury or physiologic derangements due to injury such as shock or the need for ongoing blood transfusion and fluid replacement. There were no specific exclusions for either illicit drug use or pre-existing alcoholic liver or pancreatic disease. The majority of patients also had a blood alcohol concentration determined upon admission to the hospital as part of their routine trauma evaluation. Only patients who either admitted to consuming alcohol at any time in the past year or those who had a positive blood alcohol concentration were followed for the development of AWS. Patients who claimed to be non-drinkers or had a BAC measurement of zero were not followed daily for AWS development.

Patients were initially screened on the first day of hospitalization for alcohol use using a complete Alcohol Use Disorders Identification Test (AUDIT). The AUDIT is a 10-item questionnaire designed to detect hazardous and harmful drinkers which has been validated and is extensively used in alcohol research. It assesses alcohol use in the past year characterizing it in terms of quantity, frequency, and heavy drinking episodes; symptoms of alcohol dependence; and social consequences of misuse [11-13]. Patients who consumed alcohol, as determined by AUDIT question 1, "how often do you have a drink containing alcohol", were then asked about a history of alcohol withdrawal via a series of questions (see Figure 1). The revised Clinical Institute for Withdrawal of Alcohol Scale (CIWA-Ar) was administered daily to detect symptoms of alcohol withdrawal for the first 10 days or until hospital discharge. The CIWA-Ar is a validated, standardized 10 -item questionnaire developed to assess the signs and symptoms of alcohol withdrawal, graded on severity. The signs and symptoms assessed include nausea and vomiting, tactile disturbances, tremor, auditory disturbances, paroxysmal sweats, visual disturbances, anxiety, headache, agitation and disorientation. All items except disorientation are graded on a severity scale of 0 to 7 with 0 representing normal and 7 representing the highest severity. Disorientation is graded on a scale of 0 to 4 . The maximum score on the questionnaire is 67 . A score of 8 or greater is considered sufficient to require treatment for AWS $[4,8,14]$. Although the CIWA-Ar is generally used to diagnose and guide treatment for alcohol withdrawal, for this study it was used only as a diagnostic tool to assess the daily incidence and provide a consistent and reliable diagnosis of AWS. In addition, the medical records of the patients included in the study were evaluated daily for clinical suspicion of AWS by the trauma service and for benzodiazepine administration.

\section{Outcome measures}

AWS is expressed both as a percentage of patients developing AWS (risk) and as an incidence rate in patient days. In order to find an appropriate denominator for the risk and rate of AWS it was necessary to consider three factors: (1) Although it is recognized that not all drinkers are at risk for AWS, it is not clear which drinkers in the trauma population are at risk, (2) not all people who achieve high BACs will develop AWS, and (3) some patients who drink problematically do not have a BAC determined upon admission. Hence in order to answer the question "Which trauma patients are at risk for AWS?" all drinkers were included. Because only drinkers were enrolled in the study, the entire enrolled population was considered at risk for alcohol withdrawal and hence forms the denominator of the risk and rate calculations.

In order to determine factors predictive of AWS, the open ended questions of history of alcohol withdrawal were evaluated in their association to an actual diagnosis of alcohol withdrawal. If patients reported any symptoms consistent with alcohol withdrawal to the question of, "What happens when you go a few days without drinking alcohol?" they were counted as having a history of alcohol withdrawal. Potential unwarranted medication was defined as patients who would have been treated by a previous protocol in which they would have received scheduled benzodiazepines for a positive admission BAC.

Because AWS should occur only in patients who are physiologically dependent on alcohol, the subset of dependence items from the AUDIT were evaluated for their association with AWS. The dependence items from the AUDIT [13] are "How often have you found that you were not able to stop drinking once you had started?" "How often during the last year have you failed to do what was normally expected from you because of drinking"? and "How often during the last year have you needed a first drink in the morning to get yourself going after a heavy drinking session?"

\section{Statistical power and data analysis}

The hypothesis was that the proportion of patients developing AWS would be low. From prior experience it was estimated that approximately $15 \%$ of admitted patients would develop signs and symptoms of AWS. To accurately detect a proportion of $15 \%$, it was necessary to examine 84 at-risk patients (drinkers) to have a $95 \%$ confidence interval of $9.3 \%$ to $24.7 \%$. Fisher's exact test was used for categorical variables and ANOVA and Mann-Whitney $U$ test for

\begin{tabular}{|l|}
\hline 1. Do you have a history of alcohol withdrawal? \\
\hline 2. Do you have a history of delirium tremens ("DTs")? \\
\hline 3. Do you have a history of seizures? \\
\hline 4. Do you have a history of liver disease? \\
\hline 5. Do you have a history of cirrhosis? \\
\hline 5. What happens to you if you don't drink alcohol for several days? \\
\hline
\end{tabular}

Figure 1: Questions asked to drinkers on hospital day 1. 
continuous variables. 95\% confidence intervals were calculated using a Bayesian calculator [15].

\section{Results}

One hundred and thirteen patients were screened with the AUDIT and followed through discharge or for the first 10 days of hospitalization with daily CIWA-Ar administration. There were 70 (83.3\%) men and 14 (16.7\%) women. Table 1 shows the demographic characteristics of the group. Three-quarters of patients $(74.3 \%, \mathrm{n}=84)$ reported drinking alcohol in the last year. Admission BAC measurement was unavailable in $21.2 \%(\mathrm{n}=24)$ leaving 89 patients with a measured BAC, $28 \%(\mathrm{n}=$ 25) of whom were positive. The mean and median BACs for patients with a non-zero BAC result were 187.7 and $189 \mathrm{mg} / \mathrm{dl}$ respectively. No person who denied drinking had a measurable BAC or developed AWS.

Six patients (7.1\%) answered "yes" to the question "Do you have a history of alcohol withdrawal?" One (1.2\%) reported a history of delirium tremens, two (2.4\%) reported a history of hepatitis, and two (2.4\%) reported a history of seizures, one of which was stated as due to withdrawal. No patient reported a history of cirrhosis. Only 4/84 patients reported withdrawal symptoms in response to the question "What happens to you when you don't drink for a few days?" The responses were "agitated and can't sleep" $(\mathrm{n}=1)$, seizures $(\mathrm{n}=1)$ and "I withdraw; get shaky, need a drink, get a headache" $(\mathrm{n}=2)$.

Among the 84 drinkers, 4 were suspected of developing AWS, which was confirmed in 3 by a CIWA-Ar score of 8 or more $(3.6 \%$ risk, 95\% CI 1.9-11.6\%), giving an incidence rate of 1.4 episodes per 100 patient days. All 3 were treated with benzodiazepines and none needed to be transferred to the ICU. All 3 patients developing AWS admitted to a history of AWS symptoms upon stopping drinking (tremulousness, nausea, headache, needing a drink, etc.). Table 2 shows the alcohol related characteristics of patients developing AWS (based on answers to select AUDIT questions, total AUDIT score, BAC and signs of withdrawal symptoms upon cessation of drinking) compared to drinkers who did not develop AWS while in the hospital.

BAC measurements were not performed in 24/113 patients. Of the 89 with a measured BAC, 22 were positive. BAC was measured and positive in the 3 patients who developed AWS. Implementation of a prophylaxis protocol for positive BAC, would have resulted in $88 \%$ (n $=22 / 25$ ) of BAC positive patients receiving unwarranted medication to prevent alcohol withdrawal that would not have occurred. During hospitalization all patients received either morphine sulfate or oxycodone/acetaminophen opiate analgesia on an as needed basis for their injuries.

\begin{tabular}{|l|l|}
\hline Characteristic & \\
\hline Age - years mean (SD) & $37.28(16.1)$ \\
\hline Gender (male) $\mathrm{n}(\%)$ & $70(83.3 \%)$ \\
\hline Length of stay - days mean (SD & $5.0(3.9)$ \\
\hline Admission BAC mg/dl & $69.01(119.2)$ \\
Mean (SD) & $0(0-136)$ \\
\hline Median (IQR) & $7.3(6.2)$ \\
\hline AUDIT score mean (SD) & $34(40.5 \%)$ \\
\hline AUDIT $\geq 8 \mathrm{n}(\%)$ & \\
\hline
\end{tabular}

$\mathrm{BAC}=$ Blood Alcohol Concentration

AUDIT = Alcohol Use Disorders Identification Test

$\mathrm{SD}=$ Standard Deviation

Table 1: Demographic characteristics of enrolled patients.

\begin{tabular}{|c|c|c|c|}
\hline & AWS $(n=3)$ & No AWS ( $n=81)$ & p-value \\
\hline Drinking frequency & $3(100 \%)$ & $30(37.0 \%)$ & .05 \\
\hline $\begin{array}{l}\text { Drink } 3-4 \text { or more drinks per } \\
\text { occasion }\end{array}$ & $2(66.7 \%)$ & $36(46.3 \%)$ & .45 \\
\hline Binge at least monthly & $2(66.7)$ & $34(42.5 \%)$ & .40 \\
\hline At least 1 dependence symptom & $2(66.7 \%)$ & $13(16.5 \%)$ & .08 \\
\hline AUDIT $\geq 8$ & $3(100 \%)$ & $31(38.3 \%)$ & .06 \\
\hline $\mathrm{BAC}>0$ & $3 / 3(100 \%)$ & $22 / 65(33.8 \%)$ & .045 \\
\hline $\begin{array}{l}\mathrm{BAC}^{*} \text { mean } \mathrm{mg} / \mathrm{dl} \text { (range) } \\
\mathrm{BAC}^{*} \text { median } \mathrm{mg} / \mathrm{dl}(\mathrm{IQR})\end{array}$ & $\begin{array}{l}371.7(252-477) \\
386(252-477)\end{array}$ & $\begin{array}{l}162.6(8-411) \\
15858.3-233.8)\end{array}$ & .005 \\
\hline $\mathrm{BAC}>80 \mathrm{mg} / \mathrm{dl}$ & $3 / 3(100 \%)$ & $15 / 22(68.2 \%)$ & .35 \\
\hline
\end{tabular}

AWS = Alcohol Withdrawal Syndrome Confirmed by Revised Clinical Institute for Withdrawal of Alcohol Scale.

$\mathrm{BAC}=$ Blood Alcohol Concentration

${ }^{*}$ Denotes patients with measured BAC

AUDIT - Alcohol Use Disorders Identification Test

Table 2: Drinking Characteristics of Patients Developing AWS Versus Those Who Did Not.

\section{Discussion}

This prospective study demonstrates that despite high alcohol usage among trauma patients, the incidence of alcohol withdrawal syndrome in trauma patients who use alcohol may not be as frequent as initially thought to be, and is much lower than one would expect based on previous research $[1,16,17]$. Our data demonstrates an incidence of alcohol withdrawal of $3.6 \%$ among trauma patients using alcohol. However, because a substantial number of trauma patients arrive at the hospital intoxicated, it is often assumed that those with a high blood alcohol concentration are at high risk for AWS. Prior to this study, the frequency of AWS and delirium tremens in the trauma setting had only previously been described either from registry data which was evaluated retrospectively or in small patient samples $[1,16,17]$.

These previous studies have estimated alcohol withdrawal rates as high as $31 \%$ in trauma patients deemed alcoholics by DSM criteria as well as an incidence of DTs in 5\% of alcohol-dependent patients $[1,16,17]$. This study demonstrates that most trauma patients who consume alcohol are not alcoholics/dependent drinkers and hence are not at risk for withdrawal. It also corroborates what other studies have found, i.e. that dependent drinkers are clearly at greater risk for AWS than are other non-dependent drinkers. The admission blood alcohol concentration is not sufficient to determine who will suffer from AWS.

Although in the trauma setting, identifying patients with early symptoms of AWS is critical to allow timely initiation of treatment in those who need it; it is also important to avoid unnecessary treatment and unwarranted medication of people who are not at risk for the syndrome. Presumptive prophylaxis and fixed schedule benzodiazepine dosing lead to increased drug use and increased use of other hospital tests such as CT scans and laboratory evaluations [4,711]. It is important to treat AWS due to the high mortality and largely treatable nature of delirium tremens [3,4]. A number of studies have demonstrated that symptom triggered therapy, rather than a standard prophylaxis for ASW in at-risk patients leads to shorter duration of treatment, less overall drug use, less infectious complications, and less use of CT scans and laboratory evaluations [4,7-9].

Despite the lack of empiric support for prophylaxis and the existence of literature to support the efficacy of symptom triggered therapy, there are still some trauma centers that automatically and unconditionally administer AWS prophylaxis on the basis of a positive BAC alone [6]. In this study, $88 \%$ of patients with a positive BAC would have received unnecessary prophylaxis for alcohol withdrawal had this 
Citation: Sharp B, Schermer CR, Esposito TJ, Omi EC, Ton-That H, et al (2012) Alcohol Withdrawal Syndrome in Trauma Patients: A Prospective Cohort Study. J Trauma Treat 1:128. doi:10.4172/2167-1222.1000128

Page 4 of 4

liberal policy of AWS prophylaxis been followed. One unexpected observation during the study was that daily monitoring with the CIWA-Ar and institution of symptom triggered therapy prompted early detection and treatment of mild AWS that if allowed to progress may have warranted an ICU transfer.

Limitations of the study are primarily related to its small size and the patient population. Only patients admitted to a non-ICU were studied. Although symptom triggered therapy studies have been performed in ICU populations, the findings of this study cannot be extrapolated to patients who cannot communicate due to head injury, endotracheal intubation with ventilator dependence or other factors such as those which generally mandate care in an ICU setting. Additionally, patients were only followed for 10 days after admission, with many being discharged prior to that. It is conceivable that their length of stay was short enough such that they did not undergo withdrawal while in the hospital. Therefore, AWS may have gone undetected in these patients with very short durations of stay.

An additional limitation is that we did not screen for concomitant illicit drug use and drug withdrawal during this study. Some drug withdrawal patterns may be similar to alcohol withdrawal but the primary drugs of abuse seen at our hospital are marijuana and cocaine/ crack cocaine, neither of which results in acute withdrawal symptoms similar to alcohol so we do not believe they confounded our findings. In hospitalized trauma patients and in particular in our specific hospital, problematic alcohol use occurs in a far greater percentage of patients than does illicit drug use. We have not found trauma surgeons that prophylax for withdrawal from illicit drugs based on screening and hence do not believe that patients are being over-treated for presumptive drug withdrawal.

This study should not diminish the attention given to injured patients who drink. Given the low incidence rate of AWS detected in this study, trauma surgeons should expect to see AWS in their admitted populations but not with such a frequency that it warrants routine prophylaxis. Further study is warranted to determine if particularly high BACs or other simple predictive factors such as the open ended questions used in this study can be validated and be utilized as a legitimate screening tool to prevent severe consequences of alcohol withdrawal such as seizures or delirium tremens.

\section{Acknowledgement}

Funded in part by NIAAA RO1 AA015067, Trauma Center Brief Alcohol Treatments and Cost Effectiveness, PI: T J Esposito and the Ralph and Marion Falk Foundation.

\section{References}

1. Lukan JK, Reed DN Jr, Looney SW, Spain DA, Blondell RD (2002) Risk factors for delirium tremens in trauma patients. J Trauma 53: 901-906.

2. Field CA, Claassen CA, O'Keefe G (2001) Association of alcohol use and other high-risk behaviors among trauma patients. J Trauma 50: 13-19.

3. Al-Sanouri I, Dikin M, Soubani AO (2005) Critical care aspects of alcoho abuse. South Med J 98: 372-381.

4. (2001) Committee on Practice Guidelines, Working Group on Pharmacological Management of Alcohol Withdrawal: Alcohol Withdrawal. Bethesda MD: American Society of Addiction Medicine.

5. Kosten TR, O'Connor PG (2003) Management of drug and alcohol withdrawal. N Engl J Med 348: 1786-1795.

6. Gentilello LM (2004) Alcohol and Drugs in Trauma. (5thedn), Moore, Feliciano, Mattox Eds. McGraw Hill, New York.

7. Daeppen JB, Gache P, Landry U, Sekera E, Schweizer V, et al. (2002) Symptom-triggered vs fixed-schedule doses of benzodiazepine for alcohol withdrawal: a randomized treatment trial. Arch Intern Med 162: 1117-1121.

8. DeCarolis DD, Rice KL, Ho L, Willenbring ML, Cassaro S (2007) Symptomdriven lorazepam protocol for treatment of severe alcohol withdrawal delirium in the intensive care unit. Pharmacotherapy $27: 510-518$.

9. Mayo-Smith MF (1997) Pharmacological management of alcohol withdrawal A meta-analysis and evidence-based practice guideline. American Society of Addiction Medicine Working Group on Pharmacological Management of Alcohol Withdrawal. JAMA 278: 144-151.

10. Saitz R, Mayo-Smith MF, Roberts MS, Redmond HA, Bernard DR, et al. (1994) Individualized treatment for alcohol withdrawal. A randomized double-blind controlled trial. JAMA 272: 519-523.

11. Bush K, Kivlahan DR, McDonell MB, Fihn SD, Bradley KA (1998) The AUDIT alcohol consumption questions (AUDIT-C): an effective brief screening test for problem drinking. Ambulatory Care Quality Improvement Project (ACQUIP) Alcohol Use Disorders Identification Test. Arch Intern Med 158: 1789-1795.

12. Dolman JM, Hawkes ND (2005) Combining the audit questionnaire and biochemical markers to assess alcohol use and risk of alcohol withdrawal in medical inpatients. Alcohol Alcohol 40: 515-519.

13. Saunders JB, Aasland OG, Babor TF, de la Fuente JR, Grant M (1993) Development of the Alcohol Use Disorders Identification Test (AUDIT): WHO Collaborative Project on Early Detection of Persons with Harmful Alcohol Consumption--II. Addiction 88: 791-804.

14. Sullivan JT, Sykora K, Schneiderman J, Naranjo CA, Sellers EM (1989) Assessment of alcohol withdrawal: the revised clinical institute withdrawal assessment for alcohol scale (CIWA-Ar). Br J Addict 84: 1353-1357.

15. http://www.causascientia.org/math_stat/ProportionCl.html

16. Spies CD, Neuner B, Neumann T, Blum S, Müller C, et al. (1996) Intercurrent complications in chronic alcoholic men admitted to the intensive care unit following trauma. Intensive Care Med 22: 286-293.

17. Bard MR, Goettler CE, Toschlog EA, Sagraves SG, Schenarts PJ, et al. (2006) Alcohol withdrawal syndrome: Turning minor injuries into a major problem. $J$ Trauma 61: 1441-1445. 